\title{
Validity of the Developmental Coordination Disorder Questionnaire' 07 and Its Association with Physical Activity in Young Children with Developmental Disabilities
}

\author{
Byungmo Ku, \\ ${ }^{1}$ Vanguard University of Southern California, California, United States
}

\author{
Received: August 26, 2020 \\ Accepted: December 3, 2020 \\ Published online: January 31, 2021
Keywords:
Developmental Disabilities
Motor Deficits
Parental Report Questionnaire
Physical Activity Behaviors
Young Children
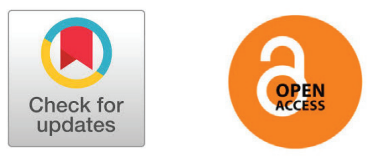

\section{ABSTRACT}

OBJECTIVES The purposes of the current study were to examine the construct and predictive validity, and reliability of the developmental coordination disorder questionnaire' 07 ( $\left(\mathrm{CDQ}^{\prime} 07\right.$ ) for young children with developmental disabilities (DD).

METHODS 135 parents of young children with DD completed an online questionnaire which consisted of demographic information, the $\mathrm{DCDQ}^{\prime} 07$, and a physical activity behavior questionnaire. A one-way analysis of variance was conducted to evaluate motor difficulties across different types of young children with DD. Exploratory factor analysis and Cronbach's alpha were used to examine construct validity and reliability of the DCDQ' 07 . Moreover, hierarchical multiple regression analysis was used to examine the association between the $\mathrm{DCDQ}^{\prime} 07$ scores and physical activity behaviors in young children with $\mathrm{DD}$.

RESULTS The one-way analysis of variance indicated that the $D_{C D Q} 07$ scores were significantly different across different types of young children with $D D=6.66, p<0.001$ ). The exploratory factor analysis revealed a three-factor solution, accounting for $64.34 \%$ of the variance of the $D_{C D Q} 07$. Internal consistencies of the three factors were acceptable. One factor, control during movement, was a significant predictor for physical activity behaviors in young children with $\mathrm{DD}(\beta=0.37, \mathrm{p}<0.01)$.

CONCLUSIONS The DCDQ' 07 may be a reliable and valid measure in examining motor difficulties in young children with DD. To promote physical activity behaviors in young children with DD, teaching and reinforcing motor skills need to be encouraged.

(๑) The Asian Society of Kinesiology and the Korean Academy of Kinesiology

\section{Introduction}

Motor skill development of children with developmental disabilities (DD) has long been a study outcome, as these children may experience a similar etiology, and motor skill deficits have been considered as one of the shared factors $[1,2]$. Children with autism spectrum disorder, aged from 5

*Correspondence: Byungmo Ku Ph.D, Division of Natural, Physical and Health Sciences, Vanguard University of Southern California, Costa Mesa, CA, 92626, USA; E-mail: Byungmo.ku@vanguard.edu
- 10 years showed significant gross motor skill delays when compared to their age matched peers [3]. Hartman et al. (2010) also found that children with mild intellectual disability had significantly lower locomotor skills compared to children without disabilities but that their objective motor skills were comparable [4]. In addition, children with cerebral palsy have lower product-oriented fundamental motor skill scores compared to children without cerebral palsy [5]. As a child develops their motor skills at a fast rate from early childhood, 
it is important to detect motor skill deficits as early as possible so that their motor skills would be improved.

The use of a parent report measure may be a way to identify motor skill deficits early in children with DD. The Developmental Coordination Disorder Questionnaire 2007 (DCDQ' 07) is a low cost and relatively convenient parent report measurement tool, designed to evaluate motor difficulties and screen developmental coordination disorder in children aged from 5 to 15 years [6]. A systematic review including 11 studies suggests that the DCDQ' 07 is one of the most promising questionnaires in examining motor difficulties in children with and without disabilities [7]. However, a missing piece of the DCDQ' 07 is that its construct validity and reliability have not been examined for parents of young children with DD.

Identifying motor skill deficits in young children with DD is important because motor skills have been considered as a prerequisite for physical activity participation, which provides variable health-related benefits $[8,9]$. A systematic review including ten articles indicates that motor skills are positively associated with habitual physical activity in children with cerebral palsy [10]. Moreover, a study conducted by Westendorp et al. (2011) found that object control skills are positively associated with organized physical activity participation in children with intellectual disabilities [11]. Children with cerebral palsy who have higher levels of motor skills are more likely to find a way to participate in physical activity [10]. In young children with DD, however, the association between motor skills and physical activity behaviors has not been widely studied. Given that physical activity provides various health-related benefits, the association should be examined.

The purpose of the current study, therefore, was to examine the construct validity and reliability of the DCDQ' 07 for young children with DD and to examine the association between DCDQ' 07 scores (parent-reported child's motor skills) and physical activity behaviors in young children with DD. It was hypothesized that the construct validity of DCDQ' 07 would be established for parents of young children with DD and that each construct of the DCDQ' 07 would be reliable. In addition, the DCDQ' 07 scores would be positively associated with physical activity behaviors in young children with DD.

\section{Methods}

\section{Subjects}

Parents of young children with DD were recruited through online social networks. It includes posting a research flyer on social media, distributing it to disability-related organizations, and sport-related programs. To participate in the present study, participants should: (a) be above 18 years in age, (b) have a child with a developmental disability, aged from 5 years to 7 years and 11 months, and (c) have been caring for the child for at least one year. Among 504 recruited participants, 135 completed an online survey which consisted of demographic information, the DCDQ' 07, and a questionnaire about physical activity behaviors of young children with DD. The Institutional Review Board at the Oregon State University approved all procedures in the current study (8706), and all participants provided informed consent.

\section{Materials}

The Developmental Coordination Disorder Questionnaire 2007

The DCDQ' 07 is a parent-reported questionnaire, designed to evaluate motor difficulties and screen developmental coordination disorder in children aged 5 - 15 years old [6]. The fundamental concept of evaluating children's motor difficulties is that parents are asked to evaluate their child's motor skills by comparing their child to another child [6]. This measure consists of 15 questions with scales ranging from $1=$ "Not at all like your child" to $5=$ "Extremely like your child". Each item's scores are summed for a total score ranged from 15 to 75 . The summed scores are used as a cutoff score, indicating possible developmental coordination disorder. The cut-off scores for children aged from 5 years to 7 years 11 months are between 15 and 46. If the summed score of a 5-year-old child is 40 , it is an indication of possible developmental coordination disorder. This tool measures three different types of motor skills: (a) control during movement (six questions), (b) fine motor (four questions), and (c) general coordination (five questions). The validity of this tool has been well supported by prior research $[6,13,14]$.

Physical activity behaviors in young children with DD 
In the current study, physical activity behaviors refer to any types of physical activity that requires more energy expenditure than rest such as brisk walking, bicycling, running, soccer, and basketball. Physical activity behaviors of young children with DD were assessed by the Modified Physical Activity Questionnaire for Children [15]. This questionnaire is a parent-proxy report, containing six items with a 5-point scale. This measure was developed based on an initial questionnaire examining children's PA participation after school time during the past 7 days [16]. Examples of this measure included but were not limited to "how often your child did physical activity (like playing sports, games, doing dance, or any other physical activity) for each day last week", "In the last 7 days, on how many right after school, did your child do sport, dance, or play games in which your child was very active?", and "In the last 7 days, on how many evenings, did your child do sport, dance, or play games in which your child was very active?”. The 2 -weeks test-retest reliability for this measure was $r=0.94$ and the convergent validity of this measure was $r=.63$ in a previous study [15]. In the current study, parents were asked to report their child's physical activity behaviors during the last 7 days and the internal consistency of this scale was

\section{Analytic Strategy}

In the present study, the two main variables had missing data: the DCDQ' 07 (0.7\%) and physical activity behaviors of young children with DD (19\%). To deal with the missing values in the survey, the Expectation-Maximization algorithm was used for the DCDQ' 07 and the multiple imputation algorithm was used for the physical activity behaviors. Multiple imputation has been widely established for replacing relatively large missingness (e.g., 10\% - 50\%) [17].

Descriptive analysis was used for demographic information of participants. To examine reliability of the DCDQ' 07, internal consistencies were calculated using Cronbach's alpha. Before conducting the exploratory factor analysis to examine the construct validity of the DCDQ' 07 , Bartlett's test of sphericity was performed to check whether the observed correlation matrix was an identity matrix. The analysis indicated that data in the current study was appropriate for the use of explanatory factor analysis ( In addition, the Kaiser-Meyer-Olkin Measure of Sampling Adequacy indicated that the strength of associations between all variables was meritorious $(\mathrm{KMO}=0.88)$. Exploratory factor analysis using a principal-component-extraction with Varimax rotation with Kaiser Normalization was used for the construct validity of the DCDQ' 07.

Pearson correlation was performed to examine correlations between different factors of the DCDQ' 07 (e.g., control during movement, fine motor, and general coordination) and physical activity behaviors in young children with DD. To examine the association between different factors of the DCDQ' 07 and physical activity behaviors in young children with $\mathrm{DD}$, controlling for covariates such as child age and parental education, hierarchical multiple regressions were performed. Three regression models were developed. The first model included control during movement as an independent variable and child's age and parent's education as covariates. The second model incorporated the first model and added fine motor as another independent variable. Finally, the third model incorporated the second model and added general coordination. All statistical analysis was computed using SPSS. 24 (SPSS Inc., Chicago, III., USA) with an alpha level of $\mathrm{p}$ $<0.05$.

\section{Results}

Data from 135 parents of children with DD was analyzed. The average age of young children with DD was 78.20 (SD: $10.44)$ months and $61.5 \%$ of children $(n=135)$ were boys with DD. The disability types included but were not limited to autism spectrum disorder (50.4\%), attention deficit/ hyperactivity disorder (10.4\%), Down syndrome (4.4\%), cerebral palsy (8.9\%), and Prader Willi syndrome (6.7\%). The remaining $19.3 \%$ of disabilities were not categorized in the disability types and they are labeled as others. $54.8 \%$ of parents of young children with DD reported that they were living in an urban area. $78.5 \%$ of parents of young children with DD were educated at least at a college level. Table 1 includes all demographic information of young children with DD. 


\section{The DCDQ' 07 Score}

The average score of the DCDQ' 07 was 34.29 (SD: 12.43). The results of ANOVA revealed that there were differences in the DCDQ' 07 scores of young children with DD by child's disability type $=6.66, p<0.001$; see Fig 1.). Post hoc comparisons using the Tukey HSD test indicated that the mean score of the DCDQ' 07 for young children with attention deficit/hyperactivity disorder $(\mathrm{M}=46.68, \mathrm{SD}=12.55)$ was significantly higher compared to young children with autism spectrum disorder $(\mathrm{M}=34.81, \mathrm{SD}=12.31)$, cerebral palsy $(\mathrm{M}$ $=22.00, \mathrm{SD}=6.46)$, and Prader Willi syndrome $(\mathrm{M}=30.11$, $\mathrm{SD}=6.31)$.

However, there was no significant difference in the DCDQ' 07 scores between young children with attention deficit/hyperactivity disorder and young children with Down syndrome. Moreover, the mean score of the DCDQ' 07 for young children autism spectrum disorder was significantly higher compared to young children with cerebral palsy. However, there was no significant difference in the DCDQ' 07 scores between young children with autism spectrum disorders and young children with Down syndrome and Prader Willie syndrome. There was no significant difference in the DCDQ' 07 score of young children with DD by child sex, living area, or parental education. The average score for physical activity behaviors in young children with DD was 2.62 (SD: 1.01) and there was no difference in the score by demographic information $<$ see Table $1>$.

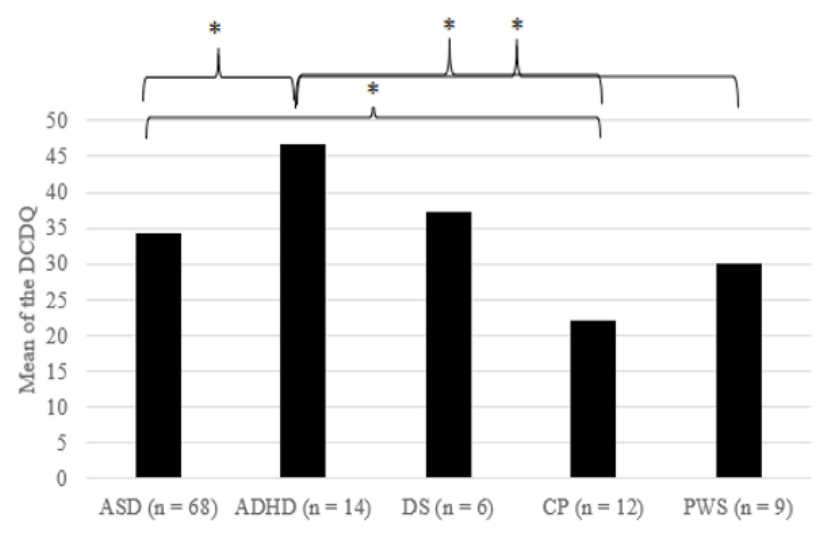

Figure 1. Average scores of the DCDQ by dissability types. Note: $\mathrm{ASD}=$ autism spectrum disorder, $\mathrm{ADHD}=$ attention deficit hyperactivity disorder, DS = down syndrome, PWS = Prader Willi Syndrome, DCDQ = developmental coordination disorder questionnaire $2007,{ }^{*} p<.05$
Table 1. Average scores of the DCDQ and physical activity behaviors by child sex, disability type, living area, and parental education in young children with DD

\begin{tabular}{lll}
\hline & Total DCDQ (SD) & $\begin{array}{l}\text { Total PA } \\
\text { (SD) }\end{array}$ \\
\hline Total & $34.29(12.43)$ & $2.62(1.01)$ \\
Child sex & & \\
Male $(n=83)$ & $33.41(12.50)$ & $2.69(1.07)$ \\
Female $(n=52)$ & $35.70(12.32)$ & $2.51(0.89)$ \\
Disability type & & \\
ASD $(n=68)$ & $34.81(12.31)$ & $2.79(1.07)$ \\
ADHD $(n=14)$ & $46.68(12.55)$ & $2.72(0.93)$ \\
DS $(n=6)$ & $37.16(7.80)$ & $2.89(0.43)$ \\
CP $(n=12)$ & $22.00(6.46)$ & $2.24(1.18)$ \\
PWS $(n=9)$ & $30.11(6.31)$ & $2.07(0.61)$ \\
Others $(n=26)$ & $32.71(11.29)$ & $2.44(0.91)$ \\
Living area & & \\
Urban $(n=74)$ & $34.93(11.31)$ & $2.58(0.94)$ \\
Rural $(n=54)$ & $32.82(13.20)$ & $2.66(1.12)$ \\
Don't want to answer $(n=5)$ & $41.60(19.69)$ & $2.81(1.10)$ \\
Parental Education & & \\
Middle school $(n=2)$ & $41.5(16.26)$ & $2.62(1.26)$ \\
High school $(n=24)$ & $35.66(12.05)$ & $2.74(1.06)$ \\
College $(2$ years, $n=44)$ & $36.39(14.32)$ & $2.72(1.02)$ \\
College $(4$ years, $n=28)$ & $31.50(10.94)$ & $2.25(0.76)$ \\
More than college level $(n=34)$ & $32.25(11.29)$ & $2.65(1.10)$ \\
Don't want to answer $(n=3)$ & $37.00(7.93)$ & $3.39(0.97)$ \\
\hline & & \\
\hline
\end{tabular}

Note. $\mathrm{DCDQ}=$ developmental coordination disorder questionnaire 2007, PA = physical activity, ASD = autism spectrum disorder, ADHD = attention deficit/hyperactivity, DS = Down syndrome, $\mathrm{CP}=$ cerebral palsy, PWS $=$ Prader Willi syndrome.

\section{Exploratory Factor Analysis}

Exploratory factor analysis revealed a three-factor solution, accounting for $64.34 \%$ of the variable with Eigenvalues of 6.76 , $1.64,1.25$ for factors 1,2 , and 3, respectively. Thus, the three factors were retained based on the Kaiser criterion [18]. The total variance of the DCDQ' 07 was explained by factor 1 (45.07\%), factor 2 (10.93\%), and factor 3 (8.33\%). Eight items loaded on factor 1 (control during movement), four items loaded on factor 2 (fine motor), and three items loaded on factor 3 (general coordination). Factor loadings of the DCDQ' 07 are listed in $<$ Table $2>$. With the small sample size of the current study, 0.6 was used as a factor loading cutoff for "good" [19]. All loaded items were considered "good", except item number 13. Cronbach's alpha for the total scores of the DCDQ' 07 was 0.90 and Cronbach's alphas for factor 1, factor 2, and factor 3 were $0.85,0.90$, and 0.68 , respectively.

Based on the results of the factor analysis, individual items of each factor were summed to be used as an independent 
Table 2. Factor loadings of explanatory factor analysis

\begin{tabular}{|c|c|c|c|}
\hline & Factor 1 & Factor 2 & Factor 3 \\
\hline \multicolumn{4}{|l|}{ DCDQ' 07 questionnaire item } \\
\hline 1. Your child throws a ball in a controlled and accurate fashion. & 0.76 & & \\
\hline 2. Your child catches a small ball (e.g., tennis ball size) thrown from a distance of 6 to 8 feet ( 1.8 to 2.4 meters). & 0.79 & & \\
\hline 3. Your child hits an approaching ball or birdie with a bat or racquet accurately. & 0.68 & & \\
\hline 4. Your child jumps easily over obstacles found in garden or play environment. & 0.69 & & \\
\hline 5. Your child runs as fast and in a similar way to other children of the same gender and age. & 0.66 & & \\
\hline $\begin{array}{l}\text { 6. If your child has a plan to do a motor activity, he/she can organize his/her body to follow the plan and } \\
\text { effectively complete the task (e.g., building a cardboard or cushion "fort," moving on playground equipment, } \\
\text { building a house or a structure with blocks, or using craft materials). }\end{array}$ & 0.64 & & \\
\hline $\begin{array}{l}\text { 7. Your child's printing or writing or drawing in class is fast enough to keep up with the rest of the children in the } \\
\text { class }\end{array}$ & & 0.79 & \\
\hline $\begin{array}{l}\text { 8. Your child's printing or writing letters, numbers and words is legible, precise and accurate or, if your child is not } \\
\text { yet printing, he or she colors and draws in a coordinated way and makes pictures that you can recognize. }\end{array}$ & & 0.89 & \\
\hline $\begin{array}{l}\text { 9. Your child uses appropriate effort or tension when printing or writing or drawing (no excessive pressure or } \\
\text { tightness of grasp on the pencil, writing is not too heavy or dark, or too light). }\end{array}$ & & 0.84 & \\
\hline 10. Your child cuts out pictures and shapes accurately and easily. & & 0.76 & \\
\hline 11. Your child is interested in and likes participating in sports or active games requiring good motor skills. & 0.68 & & \\
\hline $\begin{array}{l}\text { 12. Your child learns new motor tasks (e.g., swimming, rollerblading) easily and does not require more practice or } \\
\text { time than other children to achieve the same level of skill. }\end{array}$ & 0.78 & & \\
\hline 13. Your child is quick and competent in tidying up, putting on shoes, tying shoes, dressing, etc. & & & 0.43 \\
\hline $\begin{array}{l}\text { 14. Your child would never be described as a "bull in a china shop" (that is, appears so clumsy that he or she might } \\
\text { break fragile things in a small room). }\end{array}$ & & & 0.81 \\
\hline 15. Your child does not fatigue easily or appear to slouch and "fall out" of the chair if required to sit for long periods. & & & 0.69 \\
\hline Percentage of Variance & 45.07 & 10.93 & 8.33 \\
\hline Eigenvalue & 6.76 & 1.64 & 1.25 \\
\hline
\end{tabular}

factor for the hierarchical regression analysis. Even though items 11 and 12 loaded on factor 1 (coordination during movement) in the current study, they were excluded from the factor 1 because they were not originally categorized as coordination during movement in the DCDQ' 07 [6]. Thus, factor 1 included items 1, 2, 3, 4, 5, and 6; factor 2 included items $7,8,9$, and 10; and factor 3 included items 13, 14, and 15. Items 11 and 12 were not included in the correlation and regression analysis.

Correlation and regression between DCDQ' 07 scores and physical activity behaviors in young children with DD

The Pearson correlation analysis indicated that coordination during motion is significantly and positively associated with fine motor, general coordination, and physical activity behaviors in young children with DD. Fine motor was significantly and positively associated with general coordination. Fine motor and general coordination were not significantly associated with physical activity behaviors in young children with DD. All correlation coefficients between the variables are listed in $\langle$ Table $3>$.

Three regression models were developed for predicting physical activity behaviors in young children with DD. Results of the hierarchical multiple regression revealed that all models were significant. The first model, which included control during movement, child age, and parent's education, explained $9 \%$ of the variance ( The second model, which included variables from the first model and added fine motor, explained an additional $2 \%$ of the variance ( There was no 
Table 3. Correlation between variables between the different types of motor skills and physical activity behaviors in young children with DD

\begin{tabular}{lllll}
\hline & 1. & 2. & 3. & 4. \\
\hline 1. CDM & - & & & \\
2. FM & $0.59^{* *}$ & - & & \\
3. GC & $0.29^{* *}$ & $0.29^{* *}$ & - & \\
4. CPAB & $0.27^{* *}$ & 0.04 & 0.11 & - \\
\hline
\end{tabular}

Note. $\mathrm{CDM}=$ Control during movement, $\mathrm{FM}=$ Fine motor, $\mathrm{GC}=$ General coordination, $\mathrm{PA}=$ Child's physical activity behaviors, ${ }^{* *} p<.01$

change in the final model in explaining the variance when general coordination was added to the second model In addition, the results of the hierarchical multiple regression revealed that only control during movement was a significant predictor for physical activity behaviors of young children with DD in the three models. In all regression models, the variables fine motor and general coordination did not predict the physical activity behaviors of young children with DD. Details of the results are listed in the $<$ Table $4>$.

\begin{tabular}{|c|c|c|c|c|}
\hline \multirow{3}{*}{ Variable } & \multicolumn{4}{|c|}{ Physical activity behaviors of young children with DD } \\
\hline & \multirow{2}{*}{ Model $1 \beta$} & \multirow{2}{*}{$\begin{array}{c}\text { Model } \\
2 \beta\end{array}$} & \multicolumn{2}{|c|}{ Model 3} \\
\hline & & & $\beta$ & $95 \% \mathrm{Cl}$ \\
\hline Child age & -0.11 & -0.09 & -0.09 & {$[-0.02,0.007]$} \\
\hline $\begin{array}{l}\text { Parent's } \\
\text { education }\end{array}$ & -0.09 & -0.09 & -0.08 & {$[-0.018,0.06]$} \\
\hline $\begin{array}{l}\text { Control } \\
\text { during } \\
\text { movement }\end{array}$ & $0.27^{* *}$ & $0.37^{* *}$ & $0.36^{* *}$ & {$[0.28,0.10]$} \\
\hline Fine motor & & -0.16 & -0.17 & {$[-0.08,0.006]$} \\
\hline $\begin{array}{l}\text { General } \\
\text { coordination }\end{array}$ & & & 0.05 & {$[-0.05,0.09]$} \\
\hline$R^{2}$ & 0.09 & 0.11 & 0.11 & \\
\hline$F$ & $4.61^{* *}$ & $4.18^{* *}$ & $3.39 * *$ & \\
\hline$\Delta R^{2}$ & & 0.2 & 0 & \\
\hline$\Delta F$ & & -0.43 & -0.79 & \\
\hline
\end{tabular}

\section{Discussion}

The construct of the DCDQ' 07 was established for parents of young children with DD, with the exploratory factory analysis revealing a three-factor solution (control during movement, fine motor, and general coordination). These results are consistent with previous studies suggesting that the DCDQ' 07 provides a three-factor solution across different cultures and different populations $[6,20-22]$. Specifically, in the present study, the three-factor solution accounted for $64.34 \%$ of the variance, indicating factor stability. This result implies that the DCDQ' 07 may be a valid tool in examining three different factors of motor difficulties in young children with DD.

This result, however, should be interpreted with caution because factor loadings of the exploratory factor analysis in the present study revealed a result inconsistent with previous results $(20,21,23,24)$. In the DCDQ' 07 developed by Wilson et al. (2009), items 'like sports'(item 11) and 'learning new skills' (item 12) are categorized into the factor general coordination [6], but in the current study, the two items loaded on the factor control during movement. More research should be done to examine the difference, but a possible explanation may be found in Rivard's study [22] provided results similar to the current study. In Rivard's study (2014), the two items 'like sports' (item 11) and 'learning new skills' (item 12) also loaded on the factor control during movement. The Rivard's study suggests that the item 'learning new skills' may have loaded on the factor control during movement because of the provided examples in item 12 . The authors indicated that the examples might narrow parents' interpretation of the item. Although item 12 measures the general coordination of a child, it includes specific examples of motor tasks such as swimming and rollerblading. If the examples are excluded from the item or expanded, it may allow parents to evaluate their child's general coordination by broadening their perception. It is important to note, however, that this same reasoning does not apply to item 11, which does not include any specific examples. More research is warranted to examine why item 11 loaded on the factor control during movement for parents of young children with DD.

The reliability of the DCDQ' 07 for parents of young children with DD seems to be acceptable based on the internal consistency results of the three factors. The Cronbach's alphas for factor 1 (control during movement), factor 2 (fine motor), 
and factor 3 (general coordination) were $0.85,0.90$, and 0.68 , respectively. Compared to factors 1 and 2, factor 3 showed relatively lower internal consistency. Thus, developing more reliable items to evaluate general coordination in young children with DD should be a topic for further research. In addition, the specific definition of general coordination needs to be provided in order to develop the items.

The current study also found that there was a significant association between DCDQ' 07 scores and physical activity behaviors in young children with DD. Specifically, control during movement was a consistent significant predictor for physical activity behaviors in young children with DD across all three regression models. The final regression model, which included three factors (control during movement, fine motor, general coordination) as independent variables and child age and parental education as covariates, explained $11 \%$ of physical activity behaviors in young children with DD. In this model, control during movement alone was a significant predictor for physical activity behaviors in young children with DD $(\beta=0.36, p<0.01)$. Previous studies support this result by suggesting that motor skills are a prerequisite for physical activity participation in young children with and without disabilities $[5,9,11]$. A study conducted by Capio et al. (2012) examined five fundamental motor skills in children with cerebral palsy: running, jumping, kicking, throwing, and catching [5]. The five fundamental motor skills were significantly and positively associated with physical activity on weekdays and weekends in children with cerebral palsy. Another study also found a positive association between object-control skills such as catching, throwing, and hitting and sports participation in children with intellectual disabilities [11]. This association was also found in the longitudinal study. The motor skills of children without disabilities at 12 years of age were a significant predictor of their physical activity level (all intensity) 6 years later [25]. As control during movement measures a child's gross motor skills such as running, jumping, hitting, and catching, it logically makes sense that control during movement is a predictor of physical activity behaviors in young children with DD as well. The fundamental explanation for the association is that if a child has a better motor skill, they may be able to find more opportunities to be engaged in physical activity and/or sports compared to a child with poor motor skills.

In the current study, the average DCDQ' 07 score was 34.29 (SD: 12.43), meaning that young children with DD in the current study may experience developmental coordination disorder. As instructed by Wilson et al. (2009), if children aged from 5 years to 7 years and 11 months are in between 15 and 46 (the total DCDQ' 07 score) [6], it is an indication of possible developmental coordination disorder. This result is consistent with previous studies indicating that young children with DD experience motor skill deficits [26-29]. However, this result should be interpreted with caution because the DCDQ' 07 alone is unable to determine developmental coordination disorder in children [6]. The DCDQ' 07 is a starting point for further objective examinations such as the Movement Assessment Battery for Children-Second Edition [30] or the Bruininks-Oseretsky Test of Motor Proficiency-Second Edition [31].

Noting limitations of the current is noteworthy. Construct validity and reliability of the DCDQ' 07 should be examined in a larger number of parents of young children with DD. Based on the rule of thumb criteria [32], over 300 participants are needed to be considered 'good'. In the current study, the number of participants was only 135 . This might be a reason why items 11 and 12 loaded on control during movement, rather than on general coordination. In addition, the physical activity behaviors of young children with DD were measured by their parents. Thus, the measure may not fully represent the physical activity behaviors in young children with DDs. Future research is warranted to objectively measure the physical activity behaviors of young children with DD using accelerometers or pedometers.

\section{Conclusion}

Identifying motor difficulties or deficits in young children with DD is important as children develop motor skills at a fast rate. Early childhood is considered an ideal time for a child's fundamental development, including motor development [33]. The DCDQ' 07 may be a way to detect motor difficulties in young children with DD early because of its efficiency. Physical 
therapists, occupational therapists, or adapted physical education teachers need to encourage parents of young children with DD to evaluate their child's motor skill using the DCDQ' 07 in their daily routine. This would be a starting point to detect motor difficulties in young children with DD. In addition, the therapist or teacher needs to reinforce the motor skills (control during movement) of young children with DD. This would be a building block for young children with DD to be physically active.

\section{Acknowledgments}

A primary author of this manuscript would like to appreciate parents of children with developmental disabilities, who participated in this study.

\section{References}

1. Diamond A. Close interrelation of motor development and cognitive development and of the cerebellum and prefrontal cortex. Child Development. 2000; 71(1):4456.

2. Martin NC, Piek J, Baynam G, Levy F, Hay D. An examination of the relationship between movement problems and four common developmental disorders. Human Movement Science. 2010; 29(5):799-808.

3. Liu T, Hamilton M, Davis L, ElGarhy S. Gross Motor Performance by Children with Autism Spectrum Disorder and Typically Developing Children on TGMD-2. Journal of Child and Adolescent Behaviour. 2014; http://www.esciencecentral.org/journals/ gross-motor-performance-by-children-with-autismspectrum-disorder-and-typically-2375-4494.1000123. php?aid=22538. (Accessed Aug $25^{\text {th }} 2020$ )

4. Hartman E, Houwen S, Scherder E, Visscher C. On the relationship between motor performance and executive functioning in children with intellectual disabilities: Motor and executive functioning in children with ID. Journal of Intellectual Disability Research. 2010; 54(5):468-77.

5. Capio CM, Sit CHP, Abernethy B, Masters RSW.
Fundamental movement skills and physical activity among children with and without cerebral palsy. Research in Developmental Disabilities. 2012; 33(4):1235-41.

6. Wilson BN, Crawford SG, Green D, Roberts G, Aylott A, Kaplan BJ. Psychometric Properties of the Revised Developmental Coordination Disorder Questionnaire. Physical \& Occupational Therapy In Pediatrics. 2009; 29(2):182-202.

7. Asunta P, Viholainen H, Ahonen T, Rintala P. Psychometric properties of observational tools for identifying motor difficulties - a systematic review. BMC Pediatr. 2019; 19(1):322.

8. Centers for Disease Control and Prevention [CDC]. Physical activity and health. 2015; http://www.cdc.gov/ physicalactivity/basics/pa-health/index.htm. (Accessed Aug 25 $\left.{ }^{\text {th }}, 2020\right)$

9. Stodden DF, Goodway JD, Langendorfer SJ, Roberton MA, Rudisill ME, Garcia C, et al. A Developmental Perspective on the Role of Motor Skill Competence in Physical Activity: An Emergent Relationship. Quest. 2008; 60(2):290-306.

10. Keawutan P, Bell K, Davies PSW, Boyd RN. Systematic review of the relationship between habitual physical activity and motor capacity in children with cerebral palsy. Research in Developmental Disabilities. 2014; 35(6):1301-9.

11. Westendorp M, Houwen S, Hartman E, Visscher C. Are gross motor skills and sports participation related in children with intellectual disabilities? Research in Developmental Disabilities. 2011; 32(3):1147-53.

12. Ku B, MacDonald M, Hatfield B, Gunter KB. Parental influences on parent-reported motor skills in young children with developmental disabilities. Disability and Health Journal. 2020; 100910.

13. Cairney J, Missiuna C, Veldhuizen S, Wilson B. Evaluation of the psychometric properties of the developmental coordination disorder questionnaire for parents (DCD-Q): Results from a community based study of school-aged children. Human Movement Science. 2008; 27(6):932-40. 
14. Tseng M-H, Fu C-P, Wilson BN, Hu F-C. Psychometric properties of a Chinese version of the Developmental Coordination Disorder Questionnaire in communitybased children. Research in Developmental Disabilities. 2010; 31(1):33-45.

15. Leung KM, Chung P-K, Ransdell LB, Gao Y. Evaluation of the Psychometric Properties of the Parents' Proxy MPAQ-C in Chinese Population. Meas Phys Edu Exerc Sci. 2016; 20:112-20.

16. Crocker PR, Bailey DA, Faulkner RA, Kowalski KC, McGrath R. Measuring general levels of physical activity: preliminary evidence for the Physical Activity Questionnaire for Older Children. Med Sci Sports Exerc. 1997; 29(10):1344-9.

17. Zhang P. Multiple Imputation: Theory and Method. International Statistical Review. 2007; 71(3):581-92.

18. Kaiser HF. The Application of Electronic Computers to Factor Analysis. Educ Psychol Meas. 1960; 20(1):14151.

19. MacCallum RC, Widaman KF, Zhang S, Hong S. Sample size in factor analysis. Psychological Methods. 1999; 4(1):84-99.

20. Ferreira L, Gabbard C, Vieira JL, Tamplain P. Associations Between the Developmental Coordination Disorder Questionnaire - Brazilian Version (DCDQ-BR) and Motor Competence in School-Age Children. Phys Occup Ther Pediatr. 2020; 40(2):121-33.

21. Hua J, Gu G, Zhu Q, Wo D, Liu M, Liu J-Q, et al. The reliability and validity of the Developmental Coordination Disorder Questionnaire’07 for children aged $4-6$ years in mainland China. Res Dev Disabil. 2015; 47:405-15.

22. Rivard L, Missiuna C, McCauley D, Cairney J. Descriptive and factor analysis of the Developmental Coordination Disorder Questionnaire (DCDQ`07) in a population-based sample of children with and without Developmental Coordination Disorder: Descriptive and factor analysis of DCDQ. Child Care Health Dev. 2014; 40(1):42-9.

23. Caravale B, Baldi S, Gasparini C, Wilson BN. Crosscultural adaptation, reliability and predictive validity of the Italian version of Developmental Coordination Disorder Questionnaire (DCDQ). European Journal of Paediatric Neurology. 2014; 18(3):267-72.

24. Kennedy-Behr A, Wilson BN, Rodger S, Mickan S. Crosscultural adaptation of the developmental coordination disorder questionnaire 2007 for German-speaking countries: DCDQ-G. Neuropediatrics. 2013; 44(5):24551.

25. Jaakkola T, Yli-Piipari S, Huotari P, Watt A, Liukkonen J. Fundamental movement skills and physical fitness as predictors of physical activity: A 6-year followup study: Motor skills, fitness, and physical activity. Scandinavian Journal of Medicine \& Science in Sports. 2016; 26(1):74-81.

26. Capio CM, Mak TCT, Tse MA, Masters RSW. Fundamental Movement Skills and Balance of Children with Down Syndrome. Journal of Intellectual Disability Research. 2018; 62(3):225-36.

27. Finn K, Válková H. Motor skill development in preschool children with mental and developmental disorders - the difference after a one year comprehensive education program. 2007; 37(4):9.

28. MacDonald M, Lord C, Ulrich DA. Motor Skills and Calibrated Autism Severity in Young Children with Autism Spectrum Disorder. Adapted Physical Activity Quarterly. 2014; 31(2):95-105.

29. Provost B, Lopez BR, Heimerl S. A Comparison of Motor Delays in Young Children: Autism Spectrum Disorder, Developmental Delay, and Developmental Concerns. J Autism Dev Disord. 2007; 37(2):321-8.

30. Henderson RW. Parent-Child Interaction: Theory, Research, and Prospects. Academic Press. 2013, p 352.

31. Bruininks-Oseretsky Test of Motor Proficiency | Second Edition. 2005; / https://www.pearsonassessments. com/store/usassessments/en/Store/ProfessionalAssessments/Motor-Sensory/Bruininks-OseretskyTest - of - Motor - Proficien cy - \% 7 C - SecondEdition/p/100000648.html. (Accessed Aug 25 th, 2020 )

32. Howard MC. A review of exploratory factor analysis decisions and overview of current practices: what we are doing and how can we improve? International Journal 
of Human-Computer Interaction. 2016; 32(1):51-62.

33. Ozmun JC, Gallahue DL. Motor development. Adapted
Physical Education and Sport (six edition). Illinois, USA, Human Kinetics. 2017, p 373 -390. 